\title{
Phenotypic transformation of smooth muscle cells from porcine coronary arteries is associated with connexin $\mathbf{4 3}$
}

\author{
XUMIN ZHANG ${ }^{*}$, XIAODONG WANG ${ }^{*}$, XIAOHUI ZHOU, XIAOYE MA, YIAN YAO and XUEBO LIU \\ Department of Cardiology, East Hospital at Tongji University, Shanghai 200120, P.R. China
}

Received February 15, 2015; Accepted December 8, 2015

DOI: $10.3892 / \mathrm{mmr} .2016 .5286$

\begin{abstract}
The current study aimed to investigate the relevance of the gap junction protein connexin $\mathrm{Cx} 43$ in coronary artery smooth muscle cell (SMC) heterogeneity and coronary artery restenosis. SMCs were isolated from the coronary artery of 3-month-old pigs using enzymatic digestion. Two distinct SMC populations were isolated: Rhomboid (R) and spindle-shaped (S) cells. S-SMCs exhibited relatively lower rates of proliferation, exhibiting a classic "hills-and valleys" growth pattern; R-SMCs displayed increased proliferation rates, growing as mono- or multi-layers. Immunofluorescent staining, polymerase chain reaction and western blotting were used to assess the expression of Cx40 and Cx43 in SMCs. For further evaluation, cultured SMCs were treated with $10 \mathrm{ng} / \mathrm{ml}$ platelet-derived growth factor (PDGF)-BB with or without the gap junction blocker $18 \alpha$-glycyrrhetinic acid. Stent-induced restenosis was assessed in vivo. Different expression patterns were observed for $\mathrm{Cx} 40$ and $\mathrm{Cx} 43$ in R- and S-SMCs. Cx40 was the most abundant $\mathrm{Cx}$ in S-SMCs, whereas CX43 was identified at relatively higher levels than $\mathrm{Cx} 40$ in R-SMCs. Notably, PDGF-BB converted S-SMCs to R-SMCs, with increased $\mathrm{Cx} 43$ expression, while $18 \alpha$-glycyrrhetinic acid inhibited the PDGF-BB-induced phenotypic alterations in S-SMCs. Additionally, restenosis was confirmed in pigs 1-month subsequent to stent placement. R-SMCs were the major cell population isolated from stent-induced restenosis artery tissues, and exhibited markedly increased Cx43 expression, in accordance with the in vitro data described above. In conclusion, the phenotypic transformation of coronary artery SMCs is closely associated with $\mathrm{Cx} 43$, which is involved in restenosis. These observations provide a basis for the use of Cx43 as a novel target in restenosis prevention.
\end{abstract}

Correspondence to: Dr Xuebo Liu, Department of Cardiology, East Hospital at Tongji University, 800 Yuntai Road, Shanghai 200120, P.R. China

E-mail: medscizxm@126.com; 1xb70@hotmail.com

${ }^{*}$ Contributed equally

Key words: coronary intervention, smooth muscle cells, connexin, restenosis

\section{Introduction}

Percutaneous coronary intervention (PCI) is breakthrough for the treatment of ischemic heart disease. It is also one of the most effective methods used in coronary disease. However, the vessel wall damage and inflammatory reaction resulting from stent placement results in restenosis and affects the long-term curative effects of PCI (1). Multiple studies have demonstrated that vascular remodeling is a complex dynamic process that is closely associated with numerous factors such as inflammation, proliferation and apoptosis of smooth muscle cells (SMCs), endothelial dysfunction and thrombosis (2). Endothelial cell damage is followed by restenosis and constitutes the pathological foundation for vascular SMC proliferation, migration, apoptosis, in addition to synthesis and degradation of the extracellular matrix (3). Restenosis subsequent to coronary artery stent implantation is a process involving intimal hyperplasia and vascular remodeling following vessel damage. The vessel smooth muscle is in the tunica media surrounded by the cell matrix, which can act as a biological and mechanical barrier and leads to smooth muscle contraction, in order to avoid movement. When the vessel is damaged, inflammatory mediators are released and induce certain types of SMCs to migrate and gather in the tunica intima (4). These cells proliferate and secrete extracellular matrix, promoting new tunica intima formation for vessel reconstruction. Vascular SMC migration is the pathological foundation of common vasculopathies including atherosclerosis, angioplasty and restenosis following coronary artery stent implantation (5). Therefore, investigation into the mechanism of SMC migration is critical to the effective treatment of vascular diseases and the prevention of restenosis subsequent to coronary artery stent implantation.

The different functions that SMCs can exert result in the presence of diverse SMC phenotypes, ranging from contractile to synthetic, which exhibit differing morphologies, expression levels of SMC marker genes, proliferative potential and migratory properties (6). The heterogeneity of vascular SMCs has been previously demonstrated (7). Contractile and synthetic SMCs represent the two ends of a spectrum of SMCs with intermediate phenotypes (8). Morphologically, contractile SMCs are elongated and spindle-shaped, whereas synthetic SMCs are less elongated (8). In addition, synthetic SMCs in general grow faster and exhibit higher levels of migratory acivity than contractile SMCs (9). 
Gap junctions are clustered channels between contacting cells through which direct intercellular communication via diffusion of ions and metabolites can occur (10). These structures exist in almost all mammalian tissues, where they predominantly mediate ion and chemical material delivery and promote coupling of different cell types $(11,12)$. Gap junctions are formed by cell-specific expression patterns of the vascular connexins Cx37, Cx40, Cx43 and Cx45 (13). Cx37 and Cx40 are expressed by vascular endothelial cells and the majority of SMCs express Cx43, with little Cx37 and Cx45 expression, with only certain SMCs expressing Cx40 (14). These observations suggest an association between these connexins and the specific properties and/or phenotypes of the SMCs, although this remains to be experimentally demonstrated.

The present study aimed to assess the relevance of $\mathrm{Cx} 43$ in the intimal hyperplasia of coronary arteries by evaluating the association between the signal transduction protein $\mathrm{Cx} 43$ and SMC phenotypic transformation in porcine coronary arteries.

\section{Materials and methods}

Porcine coronary artery samples. All animal procedures were approved by the Animal Care Committee of the East Hospital at Tongji University. A total of 15 pigs Shanghai White pigs (gender, 6 female and 9 male; age range, 3-4 months; weight, $35-40 \mathrm{~kg}$ ) were purchased from Shanghai Animal Administration Center. The stent implantation procedure was performed in the Zhongshan Hospital (Zhongshan, China). Bare metal stents [MicroPort NeuroTech (Shanghai) Co., Ltd., Shanghai, China] were implanted into the right anterior descending coronary artery, left anterior descending artery or left circumflex artery of 3-month-old pigs ( $n=5$ per group). Pigs with restenosis were confirmed by coronary angiography (15) 1 month subsequent to stent placement, and were sacrificed subsequent to anesthesia. Restenosis coronary artery samples were then collected for histology or SMC isolation from the tunica media. Control 3-month-old pigs were sacrificed by an intravenous injection of $\mathrm{KCl}$ following anesthesia with ketamine and diazepam. Subsequently, the anterior descending, circumflex and right coronary arteries were separated. Tunica media cells were isolated from the arteries and cultured.

Primary cell culture and subculture. Primary cells were isolated using tissue explantation and trypsin (Gibco; Thermo Fisher Scientific, Inc., Waltham, MA, USA) enzymatic digestion as described previously (8). SMCs were isolated into two groups $(n=6)$ and maintained in Dulbecco's modified Eagle's medium (DMEM; Gibco; Thermo Fisher Scientific, Inc.) containing 20\% fetal calf serum (FCS; Gibco; Thermo Fisher Scientific, Inc.) in a humidified environment at $37^{\circ} \mathrm{C}$ with $5 \%$ $\mathrm{CO}_{2}$. Subsequent to 3 days incubation, rhomboid-shaped cells grew from the edges of explants, in a radial pattern. Following 7 days of culture, tissue fragments were removed. The remaining SMCs reached near confluence following 7-10 days incubation, exhibiting two distinct populations under an inverted microscope (Leica DMI3000 B, Leica Microsystems, Wetzlar, Germany): Spindle-shaped and rhomboid cells as previously described (7). The two cell types were separately seeded and proliferation rates were monitored by cell counting (Scepter 2.0, Merck Millipore, Ltd., Carrigtwohill, Ireland).
Platelet-derived growth factor (PDGF) treatment of SMCs from normal coronary arteries. SMC primary cultures were maintained in DMEM supplemented with $20 \%$ FCS. At $90 \%$ confluence, cells were seeded into individual wells of 6-well tissue culture plates subsequent to trypsin digestion and incubation for $24 \mathrm{~h}$ at $37^{\circ}$. Cultured SMCs were treated with $10 \mathrm{ng} / \mathrm{ml}$ PDGF-BB (Roche Diagnostics GmbH, Mannheim, Germany) for $24 \mathrm{~h}$, followed by incubation in the presence or absence of a gap junction blocker $(100 \mu \mathrm{mol} / 1$ 18 $\alpha$-glycyrrhetinic acid; Sigma-Aldrich, St. Louis, MO, USA) for $48 \mathrm{~h}$.

Immunohistochemistry. Cultured porcine SMCs were washed twice with phosphate-buffered saline (PBS; Corning, New York, NY, USA) and fixed with $4 \%$ paraformaldehyde (Sigma-Aldrich) for $30 \mathrm{~min}$. Subsequent to incubation with primary (anti-Cx40, anti-Cx43, anti-S100A4 and anti- $\alpha$-smooth muscle actin (SMA); Abcam, Cambridge, MA, USA) and polymer helper and poly-peroxidase anti-mouse/rabbit immunoglobulin G secondary antibodies (PV-9000 kit; GBI Labs, Mukilteo, WA USA) were added for $1 \mathrm{~h}$, and diaminobenzidine (Roche Diagnostics $\mathrm{GmbH}$ ) was used for signal detection. The slides were washed under running water and tissue samples were counterstained with hematoxylin. Sections were observed and imaged using an Olympus CX31 microscope (Olympus Corporation, Tokyo, Japan).

Western blotting. Western blotting of proteins extracted from coronary artery SMCs was performed as described previously $(16,17)$. Briefly, proteins were separated by sodium dodecyl sulfate-polyacrylimide gel electrophoresis (10\%; Beyotime Institute of Biotechnology Co., Shanghai, China) and electroblotted onto nitrocellulose membranes (Merck Millipore, Ltd.). Subsequent to blocking in TBST containing 5\% w/v fat-free milk, membranes were incubated $1 \mathrm{~h}$ each with the primary antibodies rabbit anti-pig polyclonal anti-Cx43 (GJA1; cat. no., ab11370; dilution, 1:1,000; Abcam), rabbit anti-pig polyclonal anti-CX40 (GJA5; cat. no., ab38580; dilution, 1:1,000; Abcam), rabbit anti-pig polyclonal anti- $\alpha$-SMA (cat. no., ab5694; dilution, 1:1,000; Abcam), rabbit anti-pig polyclonal anti-S100A4 (cat. no., ab27957; dilution, 1:1,000; Abcam) and mouse anti-pig monoclonal anti- $\beta$-actin (cat. no. ab10024; Beijing Biosynthesis Biotechnology Co., Ltd., Beijing, China), and the HRP-conjugated goat anti-rabbit or anti-mouse secondary antibodies (cat. nos., sc-2030 or sc-2302; dilution, 1:2,000; Santa Cruz Biotechnology, Inc., Dallas, TX, USA) secondary antibody at $37^{\circ}$. The Enhanced Chemiluminescence reagent kit (Pierce Biotechnology, Inc., Rockford, IL, USA) was used for detection and the membranes were exposed to film for autoradiography (Allura xper F D 20; Philips Medical Systems Nederland B.V., Eindhoven, The Netherlands).

Immunofluorescence and hematoxylin-eosin staining. Serial cryosections (section thickness, $6 \mu \mathrm{m}$;) were obtained using Leica CM3050 S (Leica Microsystems) from coronary artery great vessels with stent implantation or normal controls. Sections were stained with hematoxylin and eosin (Beyotime Institute of Biotechnology Co.,) and examined by light microscopy.

For immunofluorescent labeling, coverslips were incubated overnight with the rabbit anti-pig polyclonal anti-Cx43, rabbit anti-pig polyclonal anti-Cx40, rabbit anti-pig polyclonal anti- $\alpha$-SMA, rabbit anti-pig polyclonal anti-S100A4 and mouse 
Table I. Primers pairs used in reverse transcription-quantitative polymerase chain reaction.

\begin{tabular}{llcr}
\hline Target & \multicolumn{1}{c}{ Primer sequences } & Length (bp) & Tm $\left({ }^{\circ} \mathrm{C}\right)$ \\
\hline CX43 & 5'-CTGAGCCCCTCCAAAGACTG-3' & 101 & 60.04 \\
& 5'-TTGTATCCGGGAGGGGACAT-3' & & 60.03 \\
CX40 & 5'-GGACAAGCTCTTCGGCTTCT-3' & 126 & 60.04 \\
& 5'-TCGCTGGTACAGGTCGAGTA-3' & & 60.04 \\
GAPDH & 5'-GGAGAACGGGAAGCTTGTCA-3' & 138 & 59.97 \\
& 5'-GCCTTCTCCATGGTCGTGAA-3' & & 60.04 \\
\hline
\end{tabular}

bp, base pairs; Tm, melting temperature; GAPDH, glyceraldehyde 3-phosphate dehydrogenase.

A
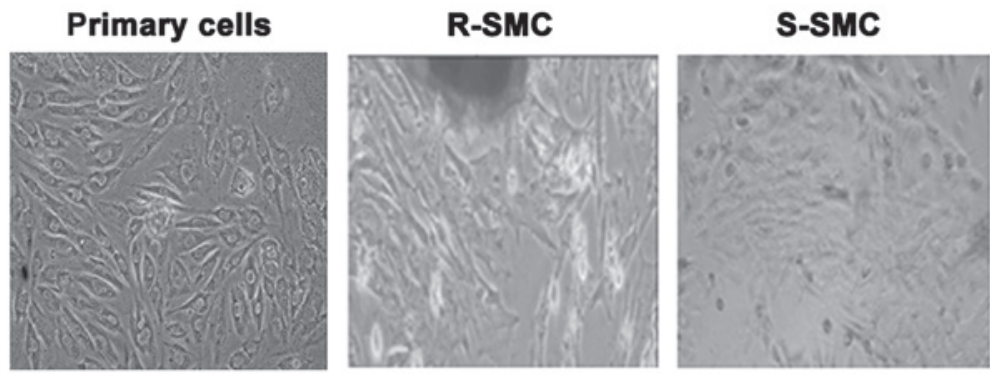

B

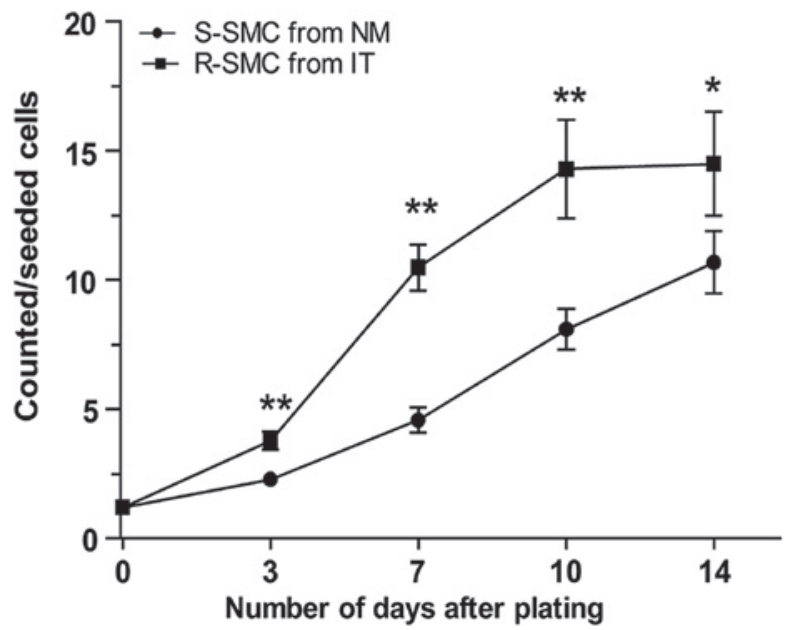

Figure 1. Two distinct SMC populations were isolated from pig coronary arteries. (A) Micrographs of SMCs isolated from pig coronary arteries. Magnification, x200 (B) Growth curves of the R-SMCs and S-SMCs in vitro. SMC, smooth muscle cell; R-SMC, rhomboid SMC; S-SMC, spindle-shaped SMC; NM, normal media; IT, intimal thickening. ${ }^{\mathrm{P}}<0.05$ and ${ }^{* *} \mathrm{P}<0.01$ vs. S-SMC from NM.

anti-pig monoclonal anti- $\beta$-actin (cat. no., ab10024; Beijing Biosynthesis Biotechnology Co., Ltd.) primary antibodies at a dilution of 1:1,000, followed by fluorescein isothiocyanate (FITC)-conjugated polyclonal goat anti-rabbit secondary antibody (cat. no. bs-0295G; Bioss Antibodies, Woburn, MA, USA) and rabbit anti-mouse secondary antibody (cat. no. bs-0368R; Bioss Antibodies) for $4 \mathrm{~h}$ at $37^{\circ}$. All steps were performed at room temperature and the cells were washed with PBS in between the steps. Cells were examined under a fluorescence microscope (FV300; Olympus Corporation) equipped with the appropriate filters.

Flow cytometry analysis. Subsequent to three washes in PBS, $1 \times 10^{6}$ SMCs were incubated with the primary antibodies for
$30 \mathrm{~min}$ at room temperature. Following another washing step cells were incubated with the FITC-labeled secondary antibody for $30 \mathrm{~min}$ at room temperature. Then, cells were washed three times with PBS and resuspended. Cell fluorescence was analyzed on a FACSCalibur flow cytometer with CellQuest software version 5.1 (BD Biosciences, Franklin Lakes, CA, USA). Gates for forward and side scatter measurements were set and a total of 10,000 events were acquired per sample.

Reverse transcription-quantitative polymerase chain reaction (RT-qPCR). Total RNA was extracted from 50-100 mg of tissue using the TRI Reagent (Molecular Research Center, Inc., Cincinnati, OH, USA); RNA concentrations were determined by spectrophotometry at $260 \mathrm{~nm}$. RT was conducted using 
A

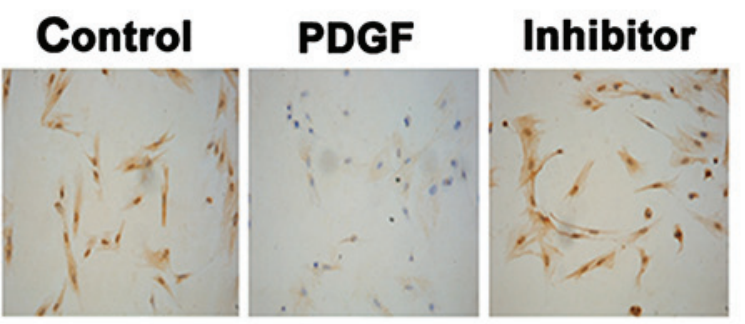

C
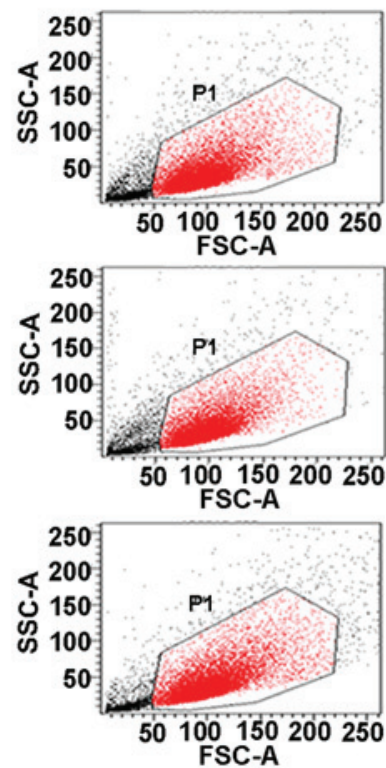

FSC-A
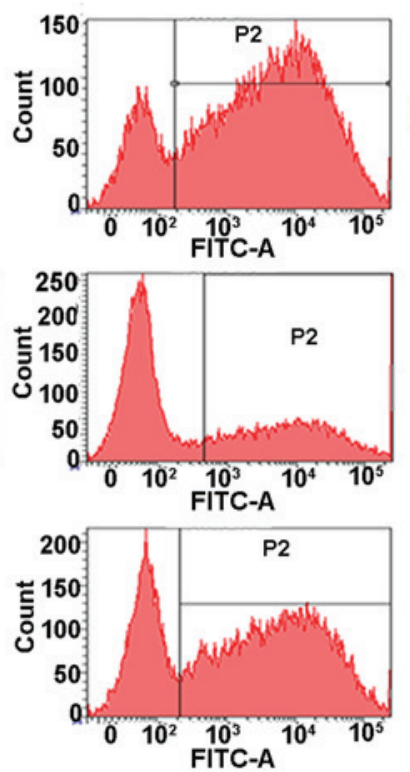

Control

B

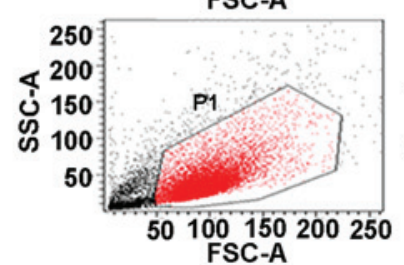

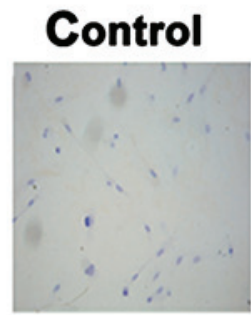

D

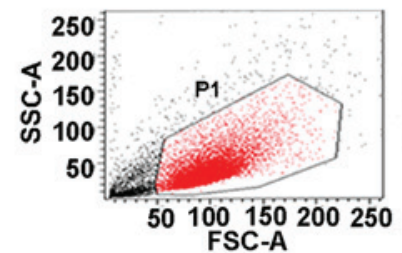

PDGF

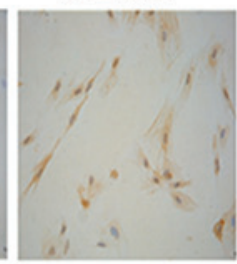

Inhibitor
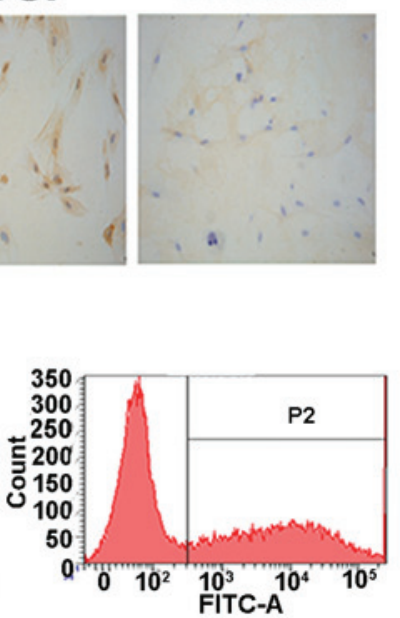

PDGF
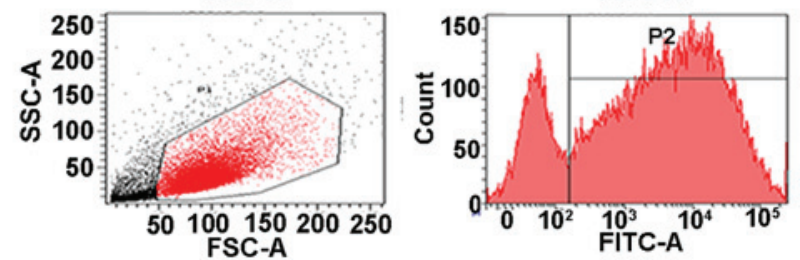

Inhibitor

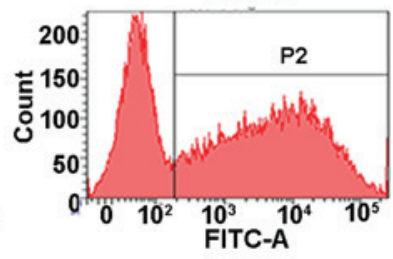

$\mathbf{E}$

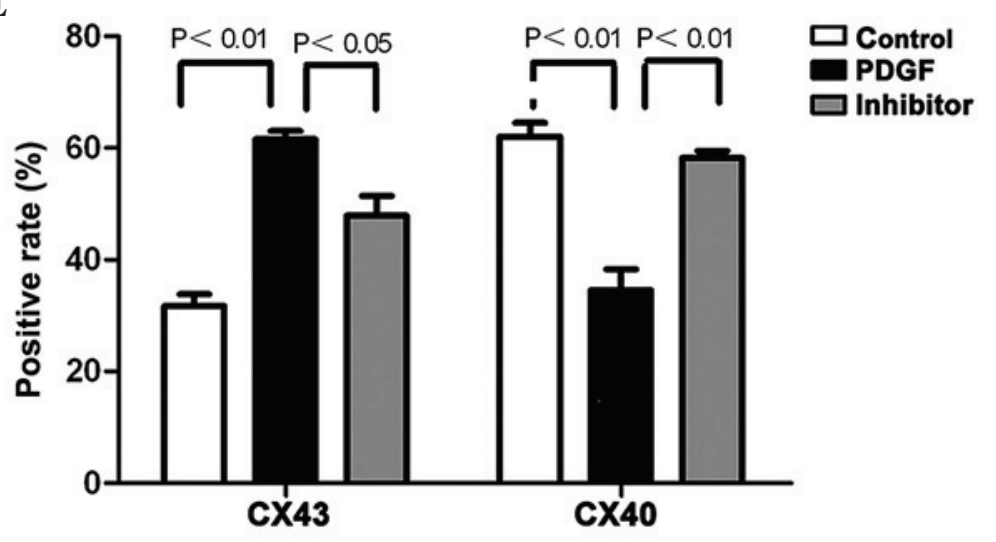

Figure 2. PDGF-induced differentiation of S-SMCs to R-SMCs is accompanied by different expression profiles of the gap junction proteins Cx40 and Cx43. Isolated S-SMCs were treated with PDGF or the gap junction blocker glycyrrhetinic acid following PDGF treatment. Immunochemical staining was used to detect the expression of (A) Cx40 or (B) Cx43 in cells of the different treatment groups. The expression levels of (C) Cx40 and (D) Cx43 in the different groups were also detected using flow cytometry. (E) Quantitative data of the flow cytometry results. PDGF, platelet-derived growth factor; SMC, smooth muscle cell; S-SMC, spindle-shaped SMC; R-SMC, rhomboid SMC; SSC, side scatter; FSC, forward scatter; FITC, fluorescein isothiocyanate.

the M-MLV1 reverse transcription kit (Promega Corporation, Madison, WI, USA). First strand cDNA was synthesized from $1 \mu \mathrm{g}$ RNA using oligo (dT) 12-18 primers. Then, RT-qPCR was performed in triplicate using FastStart Universal SYBR Green Master (Rox) from Roche Diagnostics $\mathrm{GmbH}$ for $15 \mathrm{~min}$ at $95^{\circ} \mathrm{C}$ for initial denaturation, followed by 40 cycles of $95^{\circ} \mathrm{C}$ for $30 \mathrm{sec}$ and $60 \mathrm{C}$ for $30 \mathrm{sec}$ on an ABI PRISM 7900HT Fast Real Time PCR System (Applied Biosystems; Thermo Fisher Scientific,
Inc.), according to the manufacturer's instructions. The primer pairs used are presented in Table I. The quantification of target gene relative expression was conducted using the $\Delta \mathrm{Cq}$ method (18), with glyceraldehyde 3-phosphate dehydrogenase as the internal control. All reactions were performed in triplicate.

Statistical analysis. Prism version 5.0 (GraphPad Software, Inc., San Diego, CA, USA) was used for analysis. The data are 
A

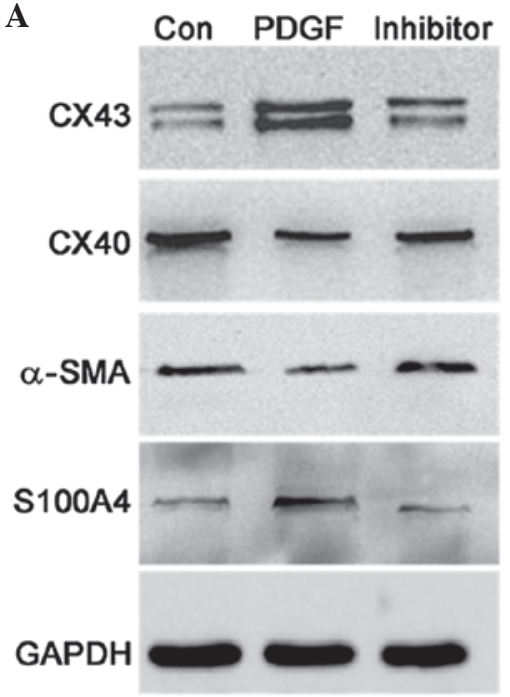

B

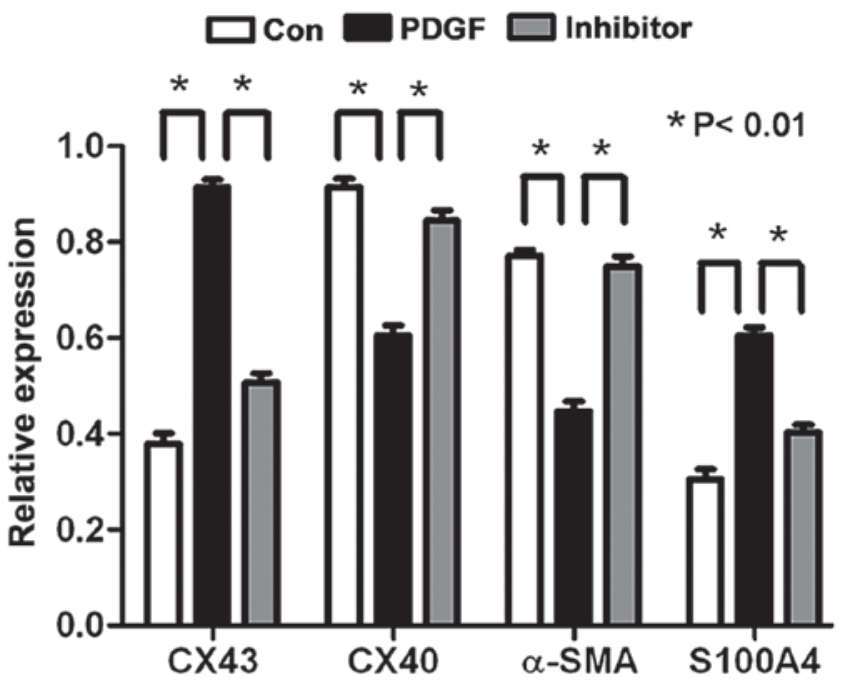

Figure 3. Expression levels of Cx40, Cx43, $\alpha$-SMA and S100A4 proteins in control, PDGF-induced or glycyrrhetinic acid blocked spindle-shaped smooth muscle cells, detected by (A) western blotting and (B) quantitative analysis. * $\mathrm{P}<0.01$. $\alpha$-SMA, $\alpha$-smooth muscle actin; PDGF, platelet-derived growth factor; GAPDH, glyceraldehyde 3-phosphate dehydrogenase.

presented as the mean \pm standard deviation and the one-way analysis of variance was used for comparisons among groups. $\mathrm{P}<0.05$ was considered to indicate a statistically significant difference.

\section{Results}

Primary culture of porcine artery cells. Subsequent to trypsin digestion, two distinct phenotypes of normal coronary artery SMCs were isolated: Rhomboid and spindle-shaped. As presented in Fig. 1, the in vitro proliferation rate was different for the two cell types. Spindle-shaped SMCs (S-SMCs), the major constituents of regular coronary artery tissue, grew in a "hills-and valleys" pattern, with a significantly lower proliferation rate. In contrast, rhomboid SMCs (R-SMCs) grew in a monolayer or multilayer pattern, with a faster proliferation rate.

The expression of gap junction proteins Cx40 and Cx43 is associated with the SMC type. The expression of two types of gap junction proteins, $\mathrm{Cx} 40$ and $\mathrm{Cx} 43$, was assessed in the isolated SMCs. In primary SMC cultures, where S-SMCs represent the majority of cells, high expression levels of Cx40 protein were detected by immunochemical staining, and this was confirmed by the amount of $\mathrm{Cx} 40$-positive cells obtained by flow cytometry (Fig. 2). Incubation with PDGF-BB induced S-SMC differentiation towards R-SMC and cells switched from a rhomboid shape to an oval shape. In agreement, a reduction in the $\mathrm{Cx} 40$ protein levels and increased $\mathrm{Cx} 43$ protein levels were observed. Notably, treatment with the Cx43 inhibitor $18 \alpha$-glycyrrhetinic acid reversed the PDGF-BB effects: R-SMC switched to S-SMC; reduced $\mathrm{Cx} 43$ protein levels and increased $\mathrm{Cx} 40$ protein levels were observed in the SMCs. Western blot analysis (Fig. 3) confirmed the reduced $\mathrm{Cx} 40$ and increased $\mathrm{Cx} 43$ protein expression subsequent to PDGF-BB treatment. Notably, PDGF-BB also reduced $\alpha$-SMA while increasing S100A4 protein expression in SMCs. All the PDGF-BB effects were reversed subsequent to treatment with the $\mathrm{Cx} 43$ inhibitor.

Stent implantation induced restenosis and SMC phenotype alterations in coronary artery tissues were associated with gap junction protein expression patterns. Using angiography technology, it was confirmed that stent implantation resulted in restenosis in the coronary artery (Fig. 4A). A comparison between restenosis and normal coronary artery tissues revealed a different composition of SMCs. In normal artery tissues, S-SMCs were the most abundant cells, whereas higher numbers of R-SMC were observed in the restenosis tissues (Fig. 4B). The expression of Cx40 and Cx43 in SMCs isolated from artery tissues was detected by flow cytometry. As presented in Fig. 5, Cx43 expression was significantly increased following stent implantation, while the levels of Cx40 expression were significantly reduced. The alterations in Cx40 and Cx43 expression subsequent to stent implantation at the protein and mRNA levels were also confirmed by western blotting and RT-qPCR (Fig. 6). In addition, the expression levels of $\alpha$-SMA and S1004A in the SMCs were assessed. Reduced $\alpha$-SMA expression and increased S1004A levels were observed subsequent to stent implantation.

\section{Discussion}

The isolation of two distinct SMC populations from normal coronary arteries have been described in the current study: S-SMCs, which displayed relatively lower proliferation rates and R-SMCs, which grew faster. These observations are in agreement with previous studies (19). R-SMCs are present in higher proportions in SMCs from stent-induced intimal thickening compared with normal tissues, suggesting that R-SMCs possess higher proliferative and migratory activities compared with S-SMCs, and are involved in arterial repair and reste- 
A
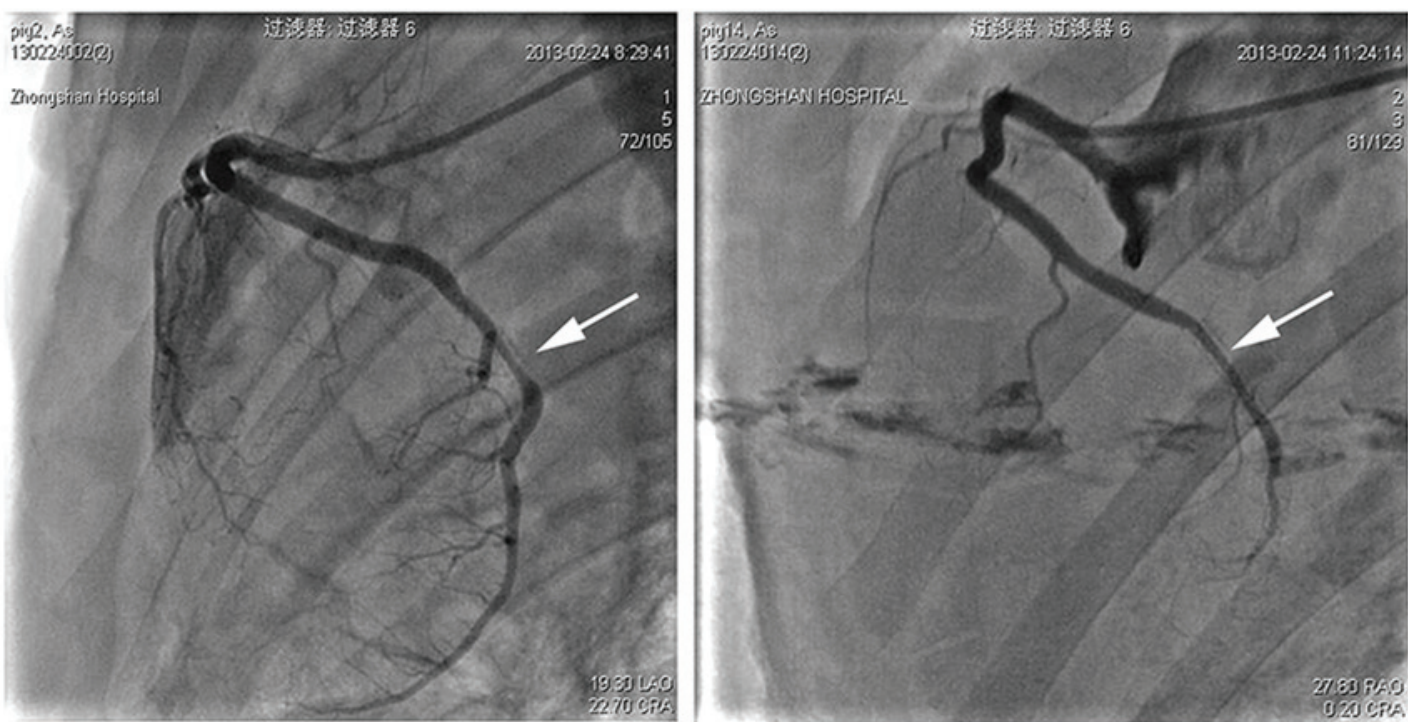

B
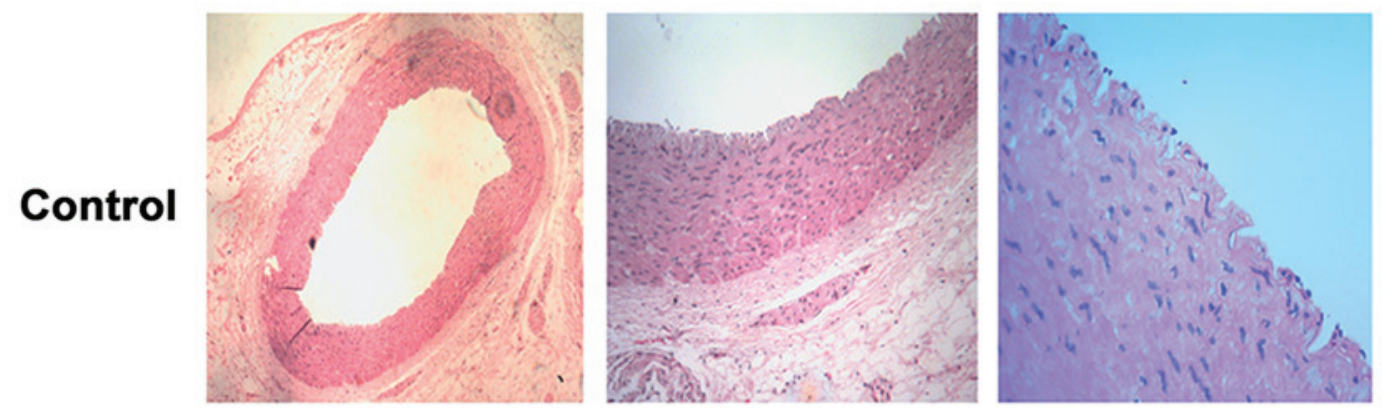

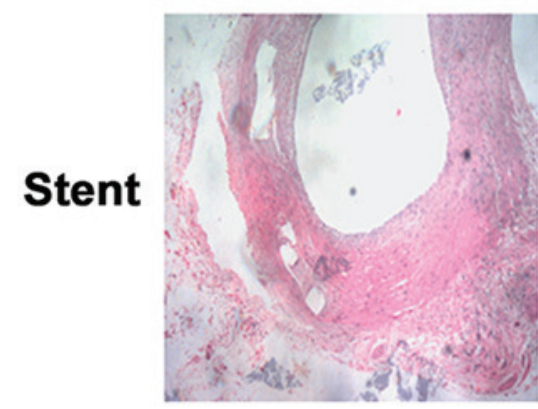

$40 \times$

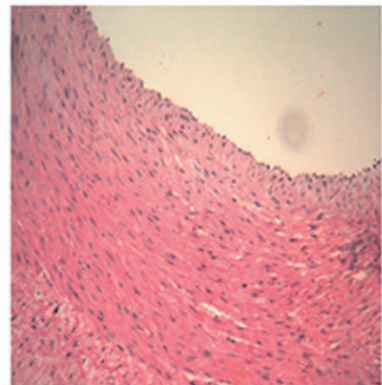

$100 x$

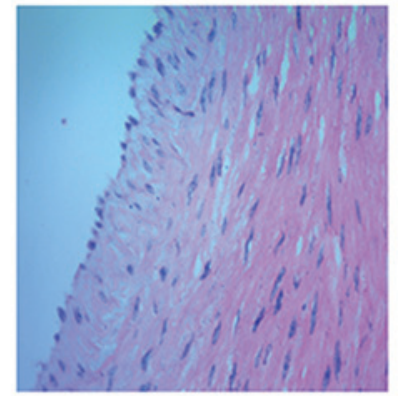

$400 x$

Figure 4. Stent implantation induced restenosis and SMC population alterations. (A) Angiography exhibiting restenosis at the stent implantation. (B) Hematoxylin and eosin staining of coronary artery tissues, demonstrating the shapes of the SMCs. SMC, smooth muscle cell.

nosis. The data of the current study corroborate with previous studies that demonstrated that fast growing R-SMCs, which display clear $\mathrm{Cx} 43$ expression and a high migratory rate (8), are present in higher proportions from stent-induced intimal thickening, suggesting that they participate in the restenotic process (19).

Cytokines, tissue factors and inflammatory factors are released subsequent to vessel damage. These factors activate SMCs in media; induce SMC phenotypic modulation, including contraction and synthesis and the secretion of extracellular matrix, which promotes SMC proliferation and migration to the intima (20-23). PDGF is an inflammatory factor and comprises several members, including PDGF-AA, PDGF-BB, PDGF-AB, PDGF-CC and PDGF-DD. PDGF is involved in cell signal transduction and transcriptional activation through the binding of PDGF membrane receptors, and it can additionally promote cell mitosis and induce SMC phenotypic modulation (24). With platelet deposition, SMC phenotypic modulation and new intima formation are enhanced by platelet-activating factors (25). Migration and proliferation of vessel SMCs are important cellular processes in the initiation stage of vascular remodeling, and are the key causes of restenosis subsequent to stent implantation (5).

Phenotypic modulation, proliferation and migration of SMCs serve a critical role in restenosis, and SMCs have been predominantly evaluated in studies describing the association between connexin and restenosis (26). The current study identified an association between phenotypic modulation of SMCs from porcine coronary arteries and the expression of connexins. Two distinct SMC populations from normal coronary arteries were isolated, S-SMCs and R-SMCs (7). In the current study, Cx43 expression was observed to be greater in R-SMCs 


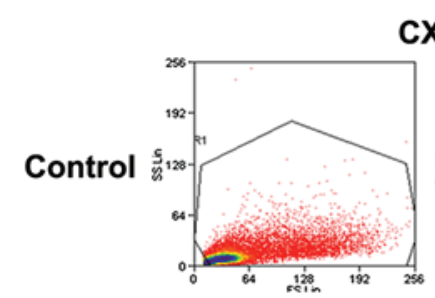

CX43

CX40
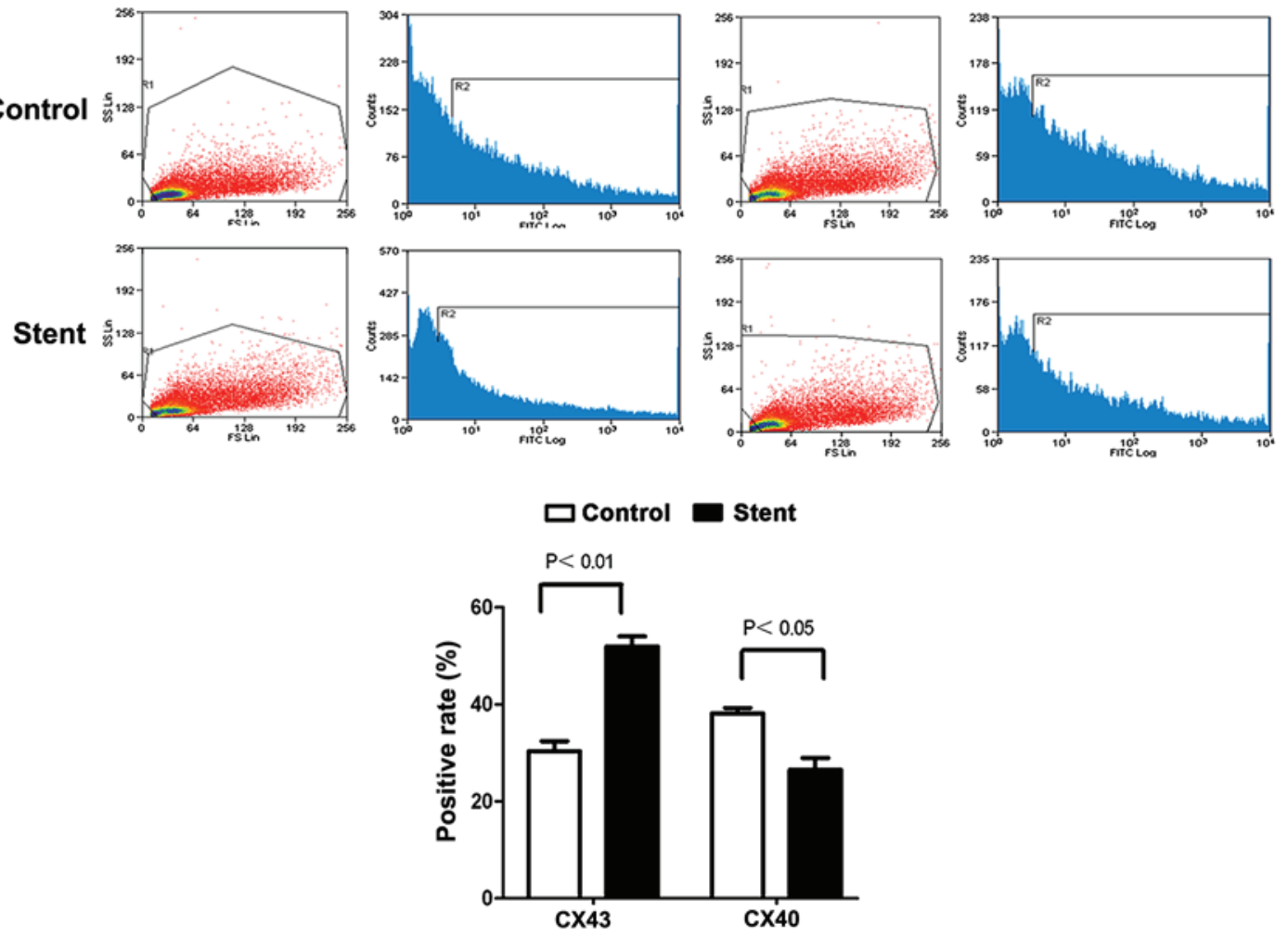

Figure 5. Stent implantation induced alterations in the SMC populations. SMCs were isolated from coronary artery tissues with restenosis and were labeled with immunochemical staining of $\mathrm{Cx} 40$ or $\mathrm{Cx} 43$. The cell populations with $\mathrm{Cx} 40$ or $\mathrm{Cx} 43$ expression were detected by flow cytometry. SMC, smooth muscle cell.
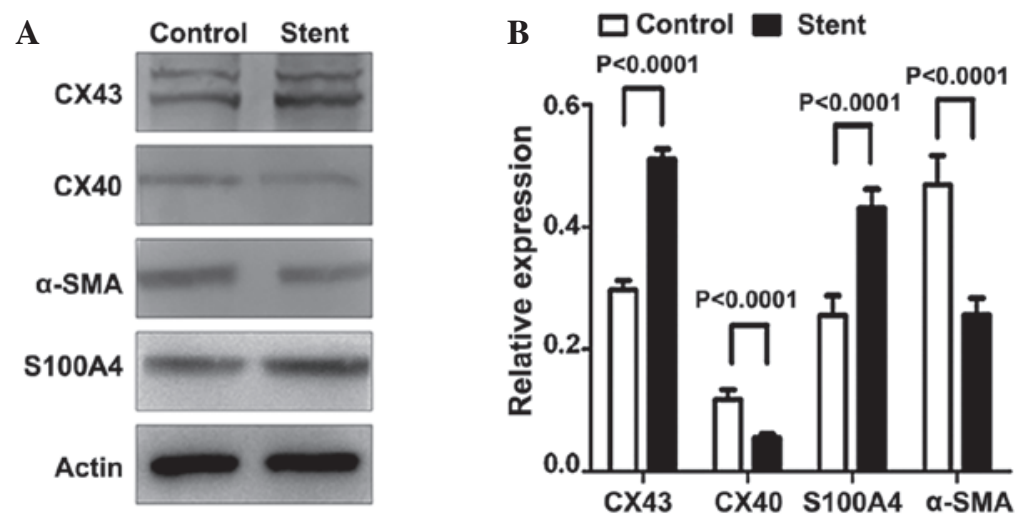

Figure 6. Stent implantation induced alterations in the expression levels of gap junction proteins in addition to $\alpha$-SMA and S100A4. (A) Isolated smooth muscle cells from coronary artery tissues were immunochemically stained for $\alpha$-SMA or S100A4. The protein levels of $\alpha$-SMA and S100A4, together with Cx40 and Cx43 were detected using western blotting, with actin as an internal control. (B) The mRNA levels of CX40, CX43, $\alpha$-SMA and S100A4 were detected using reverse transcription-quantitative polymerase chain reaction. $\alpha$-SMA, $\alpha$-smooth muscle actin.

compared with S-SMCs in vitro, indicating that Cx43 expression is phenotype-dependent in SMCs. Notably, PDGF-BB induced the transition of S-SMCs to R-SMCs, with resulting increased $\mathrm{Cx} 43$ expression. However, when $\mathrm{C} x 43$ expression is blocked by antisense RNA, the phenotypic alterations induced by PDGF-BB are reported to be reverted with the expression of $\alpha$-SMA. In accordance with previous reports $(27,28)$, the number of R-SMCs was reported to be increased and $\mathrm{Cx} 43$ expression was upregulated in the intima of restenosis subsequent to coronary artery stent implantation. These studies also revealed that inhibition of $\mathrm{Cx} 43$ expression blocks macrophage infiltration in addition to SMC proliferation and migration, which is in agreement with previous studies (29,30-32) describing the phenotypic modulation of porcine coronary arteries.

The formation of new intima was further inhibited subsequent to stent implantation. Taken together, the above results suggest that Cx43 levels are clearly associated with the SMC phenotypic and migratory patterns, suggesting that $\mathrm{Cx} 43$ may be a potential novel target for restenosis prevention.

Overall, $\mathrm{Cx} 43$ is closely associated with restenosis subsequent to PCI. Although the mechanism underlying these observations remains unclear, these observations provide a basis for the use 
of Cx43 as a molecular target to prevent restenosis subsequent to stent implantation. Downregulation of $\mathrm{Cx} 43$ expression and activity may influence the pathological process of vascular remodeling by inhibiting SMC migration at an early stage, thus improving vascular remodeling and preventing restenosis.

\section{Acknowledgements}

The present study was supported by the Scientific Committee of Pudong New District (grant no. PKJ20112-Y21).

\section{References}

1. Molica F, Burger F, Thomas A, Staub C, Tailleux A, Staels B, Pelli G, Zimmer A, Cravatt B, Matter CM, et al: Endogenous cannabinoid receptor $\mathrm{CB} 1$ activation promotes vascular smooth-muscle cell proliferation and neointima formation. J Lipid Res 54: 1360-1368, 2013.

2. Shu ZW, Yu M, Chen XJ and Tan XR: Ghrelin could be a candidate for the prevention of in-stent restenosis. Cardiovasc Drugs Ther 27: 309-314, 2013.

3. Werner N, Priller J, Laufs U, Endres M, Böhm M, Dirnagl U and Nickenig G: Bone marrow-derived progenitor cells modulate vascular reendothelialization and neointimal formation: Effect of 3-hydroxy-3-methylglutaryl coenzyme a reductase inhibition. Arterioscler Thromb Vasc Biol 22: 1567-1572, 2002.

4. Cheng XW, Kuzuya M, Nakamura K, Di Q, Liu Z, Sasaki T, Kanda S, Jin H, Shi GP, Murohara T, et al: Localization of cysteine protease, cathepsin $\mathrm{S}$, to the surface of vascular smooth muscle cells by association with integrin alphanubeta3. Am J Pathol 168: 685-694, 2006.

5. Cai X: Regulation of smooth muscle cells in development and vascular disease: Current therapeutic strategies. Expert Rev Cardiovasc Ther 4: 789-800, 2006.

6. Rensen SS, Doevendans PA and van Eys GJ: Regulation and characteristics of vascular smooth muscle cell phenotypic diversity. Neth Heart J 15: 100-108, 2007.

7. Hao H, Ropraz P, Verin V, Camenzind E, Geinoz A, Pepper MS, Gabbiani G and Bochaton-Piallat ML: Heterogeneity of smooth muscle cell populations cultured from pig coronary artery. Arterioscler Thromb Vasc Biol 22: 1093-1099, 2002.

8. Hao H, Gabbiani G and Bochaton-Piallat ML: Arterial smooth muscle cell heterogeneity: Implications for atherosclerosis and restenosis development. Arterioscler Thromb Vasc Biol 23: $1510-1520,2003$.

9. Chamley-Campbell J, Campbell GR and Ross R: The smooth muscle cell in culture. Physiol Rev 59: 1-61, 1979.

10. Willecke K, Eiberger J, Degen J, Eckardt D, Romualdi A, Güldenagel M, Deutsch U and Söhl G: Structural and functional diversity of connexin genes in the mouse and human genome. Biol Chem 383: 725-737, 2002.

11. Vinken M, Vanhaecke T, Papeleu P, Snykers S, Henkens T and Rogiers V: Connexins and their channels in cell growth and cell death. Cell Signal 18: 592-600, 2006.

12. Venance L, Glowinski J and Giaume C: Electrical and chemical transmission between striatal GABAergic output neurones in rat brain slices. J Physiol 559: 215-230, 2004.

13. Kurtz L, Madsen K, Kurt B, Jensen BL, Walter S, Banas B, Wagner $\mathrm{C}$ and Kurtz A: High-level connexin expression in the human juxtaglomerular apparatus. Nephron Physiol 116: p1-p8, 2010.

14. Shav D, Gotlieb R, Zaretsky U, Elad D and Einav S: Wall shear stress effects on endothelial-endothelial and endothelial-smooth muscle cell interactions in tissue engineered models of the vascular wall. PLoS One 9: e88304, 2014.
15. Shen L, Wu Y, Zhang F, Wu L, Dong C, Gao Y, Sun A, Zou Y, Qian J, Sun J, et al: Assessment of an asymmetrical coating stent with sirolimus released from ablumial matrix in porcine model. Clin Res Cardiol 101: 917-927, 2012.

16. Chadjichristos CE, Matter CM, Roth I, Sutter E, Pelli G, Lüscher TF, Chanson M and Kwak BR: Reduced connexin43 expression limits neointima formation after balloon distension injury in hypercholesterolemic mice. Circulation 113: 2835-2843, 2006.

17. Brisset AC, Hao H, Camenzind E, Bacchetta M, Geinoz A, Sanchez JC, Chaponnier C, Gabbiani G and Bochaton-Piallat ML: Intimal smooth muscle cells of porcine and human coronary artery express S100A4, a marker of the rhomboid phenotype in vitro. Circ Res 100: 1055-1062, 2007.

18. Livak KJ and Schmittgen TD: Analysis of relative gene expression data using real-time quantitative PCR and the 2(-Delta Delta C(T)) Method. Methods 25: 402-408, 2001.

19. Chadjichristos CE, Morel S, Derouette JP, Sutter E, Roth I, Brisset AC, Bochaton-Piallat ML and Kwak BR: Targeting connexin 43 prevents platelet-derived growth factor-BB-induced phenotypic change in porcine coronary artery smooth muscle cells. Circ Res 102: 653-660, 2008.

20. Bujo H and Saito Y: Modulation of smooth muscle cell migration by members of the low-density lipoprotein receptor family. Arterioscler Thromb Vasc Biol 26: 1246-52, 2006.

21. Thyberg $\mathrm{J}$ and Hultgårdh-Nilsson A: Fibronectin and the basement membrane components laminin and collagen type IV influence the phenotypic properties of subcultured rat aortic smooth muscle cells differently. Cell Tissue Res 276:263-271, 1994.

22. Li X, Van Putten V, Zarinetchi F, Nicks ME, Thaler S, Heasley LE and Nemenoff RA: Suppression of smooth-muscle alpha-actin expression by platelet-derived growth factor in vascular smooth-muscle cells involves Ras and cytosolic phospholipase A2. Biochem J 327: 709-716, 1997.

23. Su B, Mitra S, Gregg H, Flavahan S, Chotani MA, Clark KR, Goldschmidt-Clermont PJ and Flavahan NA: Redox regulation of vascular smooth muscle cell differentiation. Circ Res 89: 39-46, 2001.

24. Andrae J, Gallini R and Betsholtz C: Role of platelet-derived growth factors in physiology and medicine. Genes Dev 22: 1276-1312,2008.

25. Deaton RA, Gan Q and Owens GK: Spl-dependent activation of KLF4 is required for PDGF-BB-induced phenotypic modulation of smooth muscle. Am J Physiol Heart Circ Physiol 296: H1027-H1037, 2009.

26. Morel S: Multiple roles of connexins in atherosclerosis- and restenosis-induced vascular remodeling. J Vasc Res 51: 149-161, 2014.

27. de Wit C, Wolfle SE and Höpfl B: Connexin-dependent communication within the vascular wall: Contribution to the control of arteriolar diameter. Adv Cardiol 42: 268-283, 2006.

28. Yeh HI,Lupu F,DupontE and Severs NJ: Upregulation of connexin 43 gap junctions between smooth muscle cells after balloon catheter injury in the rat carotid artery. Arterioscler Thromb Vasc Biol 17: 3174-3184, 1997.

29. Li P, Liu Y, Yi B, Wang G, You X, Zhao X, Summer R, Qin Y and Sun J: MicroRNA-638 is highly expressed in human vascular smooth muscle cells and inhibits PDGF-BB-induced cell proliferation and migration through targeting orphan nuclear receptor NOR1. Cardiovasc Res 99: 185-193, 2013.

30. Nomiyama T, Nakamachi T, Gizard F, Heywood EB, Jones KL, Ohkura N, Kawamori R, Conneely OM and Bruemmer D: The NR4A orphan nuclear receptor NOR1 is induced by platelet-derived growth factor and mediates vascular smooth muscle cell proliferation. J Biol Chem 281: 33467-33476, 2006.

31. Scott RA, Paderi JE, Sturek M and Panitch A: Decorin mimic inhibits vascular smooth muscle proliferation and migration. PLoS One 8: e82456, 2013.

32. Hua Y, Dolence J, Ramanan S, Ren J and Nair S: Bisdemethoxycurcumin inhibits PDGF-induced vascular smooth muscle cell motility and proliferation. Mol Nutr Food Res 57: 1611-1618, 2013 